In Situ

Revue des patrimoines
In Situ

Revue des patrimoines

$6 \mid 2005$

Patrimoine en situation : l'Inventaire général entre histoire et prospective

\title{
Le grand inventaire
}

\section{Michel Melot}

\section{(2) OpenEdition \\ Journals}

Édition électronique

URL : http://journals.openedition.org/insitu/8797

DOI : 10.4000/insitu.8797

ISSN : $1630-7305$

Éditeur

Ministère de la culture

Référence électronique

Michel Melot, « Le grand inventaire », In Situ [En ligne], 6 | 2005, mis en ligne le 15 mai 2012, consulté le 30 avril 2019. URL : http://journals.openedition.org/insitu/8797 ; DOI : 10.4000/insitu.8797

Ce document a été généré automatiquement le 30 avril 2019.

\section{(c) (i) (3)}

In Situ Revues des patrimoines est mis à disposition selon les termes de la licence Creative Commons Attribution - Pas d'Utilisation Commerciale - Pas de Modification 4.0 International. 


\title{
Le grand inventaire
}

\author{
Michel Melot
}

\section{NOTE DE L'ÉDITEUR}

Michel Melot a été sous-directeur de l'Inventaire général de 1995 à 2003.

1 Depuis quarante ans qu'il existe, l'Inventaire général des monuments et des richesses artistiques de la France n'a pas seulement progressé, couvrant près de dix mille communes, documentant plus de vingt mille dans cinq millions de pages, trois millions de photographies, et cent mille dessins. Il est devenu ce que ses fondateurs désiraient qu'il fût. Il n'est pas une liste d'objets plus ou moins singuliers qu'on aurait oubliés, mais l'observation méthodique de l'émergence dans le monde de l'art de catégories d'objets naguère insignifiants et qui connaissent ce que Malraux appelait la «métamorphose ». André Chastel, son initiateur, plaçait le projet d'inventaire général dans cette perspective lorsqu'il écrivait : "D'abord la notion même d'œuvre d'art cesse d'être inconditionnellement suffisante et claire. Il serait insensé de méconnaître les merveilleuses propriétés de toutes les "créations » multipliées par les hommes au-delà des besoins utilitaires et de ne pas leur accorder un statut privilégié [...] Mais il s'agit de savoir comment et à quel prix elles acquièrent précisément ce pouvoir. L'histoire des variations de ces valeurs à l'intérieur du « musée imaginaire » de chaque civilisation, de chaque époque, tend irrésistiblement à se conjuguer avec l'histoire de l'art. ${ }^{1}$ " 
Figure 1

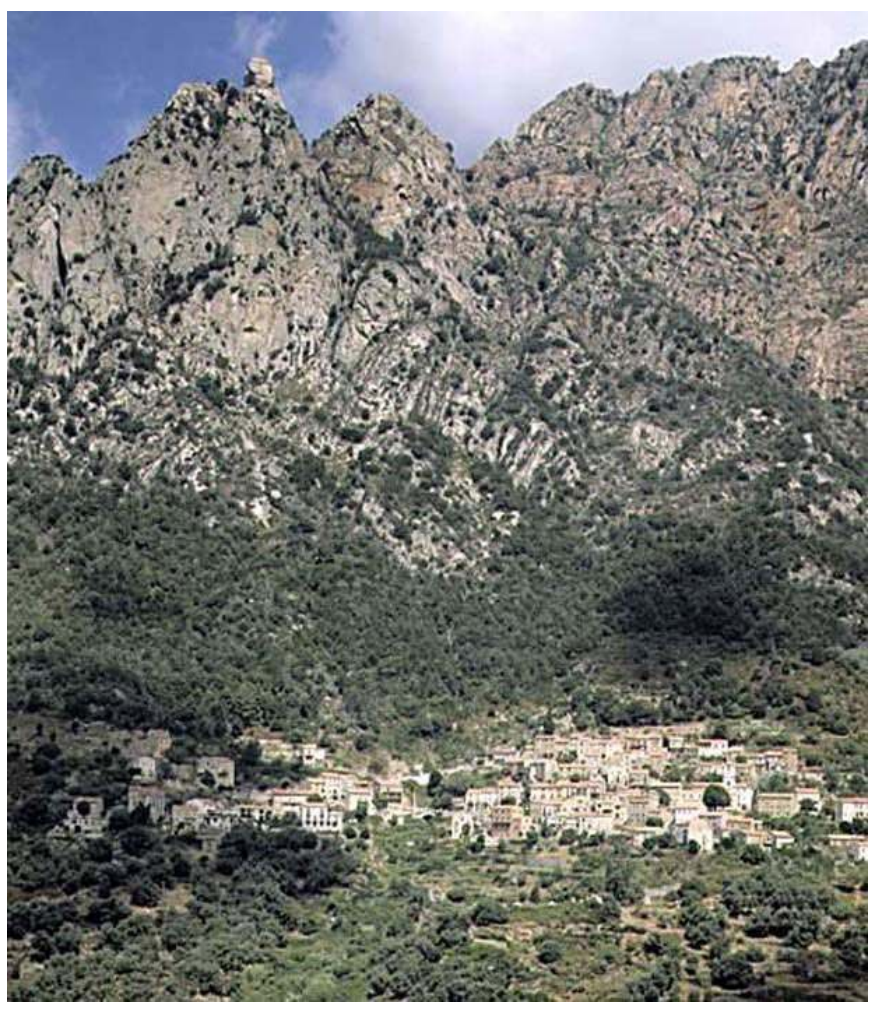

Ota (Corse-du-Sud). Vue de situation du village

Phot. Inv. J.-L. Sarrola @ Inventaire général, ADAGP, 1990

2 Nous ne reviendrons pas sur les antécédents de l'Inventaire, souvent étudiés ${ }^{2}$. Ses objectifs n'ont pas changé. Ainsi l'inventaire devra : «...guider les organisations du tourisme; assurer les tâches de l'enseignement; orienter la recherche archéologique et historique; donner enfin aux commissions responsables des monuments historiques et de l'urbanisme des éléments d'action suffisants. " ". L'originalité de l'Inventaire français tient à son caractère national: son objet, c'est la France. Il diffère des exemples étrangers en ce qu'il ne se contente pas d'être une collection de monographies. Pour A. Chastel: «Il ne peut s'agir d'épingler les notices d'édifices isolés. Tout ce qui a été dit précédemment sur les principes actuels de l'histoire de l'art amène à faire une place nouvelle aux ensembles. Il faut considérer les quartiers, les zones, le cadre urbain et, dans le meilleur des cas, le site. " (fig. $\mathbf{n}^{\circ} \mathbf{1}$ ). Cet inventaire n'en est donc pas un palmarès: on passe à une recherche globale qui s'allonge chaque jour et dont chaque ajout modifie la configuration de l'ensemble. Avec l'Inventaire français on passe de la compilation savante d'œuvres connues ou à reconnaître, à la découverte, voire à la création, de ce que l'on appellera désormais « le patrimoine ».

3 Ce mot de patrimoine qui semble aujourd'hui environner le champ de l'art et reculer ses limites jusqu'à l'infini, mérite qu'on s'y arrête 5 . Avant de désigner ce que l'on possède, le mot patrimoine qualifiait les biens inaliénables de la personne : ce que l'on est plutôt que ce que l'on $a$. Le premier patrimoine, c'est notre patronyme, notre corps ou notre image. Le reste est subsidiaire et peut être partagé. La notion de patrimoine n'a concerné les biens matériels qu'autant qu'il était nécessaire de constituer en personnes des entités collectives: familles, communautés, associations ou sociétés anonymes. La personne physique existe par elle-même, son patrimoine lui est pour ainsi dire incorporé. La 
personne morale, elle, n'existe en tant que collectivité que par ce qu'elle possède. Pas plus que la fortune, la mémoire n'est intégrée dans le groupe : il faut sans cesse la lui rendre. C'est un des rôles des inventaires ${ }^{6}$.

4 L'Inventaire pourtant est bien autre chose qu'un simple "musée imaginaire " de la France, c'est le moyen de montrer que l'art, au sens le plus large, est à l'œuvre partout dans l'activité humaine. L'adhésion de Malraux à l'idée d'un Inventaire général est exprimée dans un texte qui, en raison de sa détermination et de sa clarté, n'est pas un texte de circonstance et qu'il improvisa, avant de le réécrire, lors de l'installation de la Commission nationale de l'Inventaire, le 14 avril $1964^{7}$. Ce texte résume avec force la cohérence entre l'Inventaire et l'idée que Malraux se faisait de l'art, et doit être mis en parallèle avec le propos d'A. Chastel. «Bien que l'on rêve depuis longtemps d'une entreprise comme celle-ci, nous savons, au moment où s'ouvre cet Inventaire - destiné par la nature de nos arts à être le plus divers de tous - qu'il sera très différent de ce qu'il eût été au siècle dernier, et même lorsque furent entrepris quelques-uns des inventaires étrangers. Il apporte beaucoup plus qu'un cadastre artistique, un complément de ce qui existe dans ce domaine; le tout n'est pas seulement ici la somme de ses parties. En même temps qu'il complète nos connaissances, il suggère une mise en question sans précédent des valeurs sur lesquelles ces connaissances se fondent. »

Figure 2

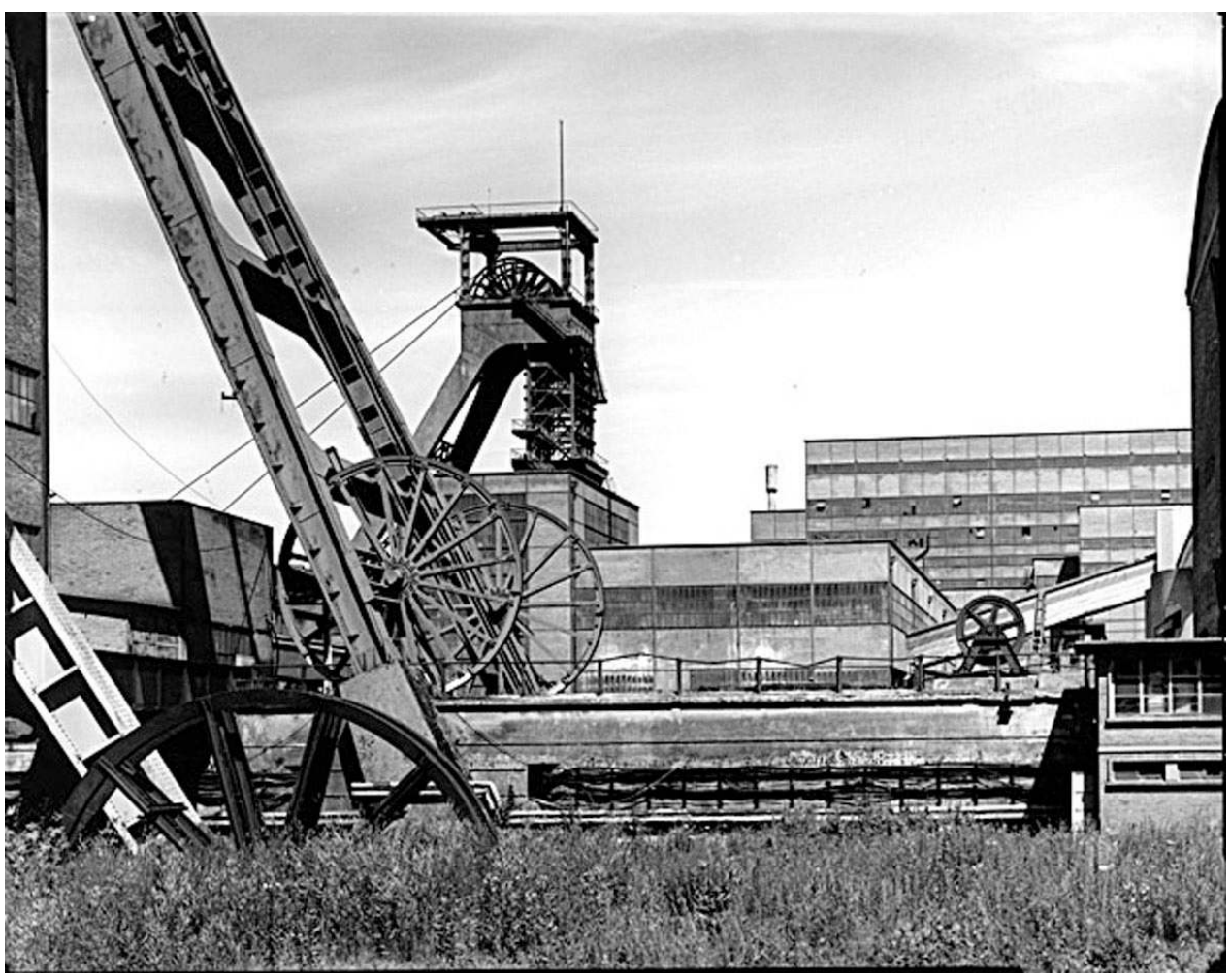

Petite-Rosselle (Moselle). Chevalement de mine

Phot. Inv. G. André (c) Inventaire général, ADAGP, 1997

5 Si le patrimoine est une notion en perpétuel devenir, est-il encore raisonnable de penser que l'Inventaire sera un jour fini? Son premier organisateur, Roger Delarozières ne le pensait déjà pas. Les inventaires réalisés voilà trente ans demandent à être révisés et complétés, tout simplement parce que notre sensibilité au patrimoine s'est avivée et que l'Inventaire en est l'instrument de mesure. Qui, en 1960, prévoyait que le patrimoine 
industriel, dont les usines fumaient encore, soit considéré comme un bien symbolique et esthétique? Qui parlait d'un patrimoine scolaire, sportif ou hospitalier (fig. $\left.\mathbf{n}^{\circ} \mathbf{2}\right)$ ? L'objet de l'inventaire étant évolutif, comment pourrait-il être une opération figée dans le temps? Le principe même en serait vicieux: les édifices, les objets d'art ne sont pas immuables. La mémoire aussi a son histoire.

Pour répondre aux injonctions de Malraux: « ... nous ne tentons plus un inventaire des formes conduit par la valeur connue : beauté, expression, etc. qui orientait la recherche ou la résurrection, mais à quelques égards le contraire, » le champ de l'Inventaire doit être sans frontières dans ses choix. Mais quels que soient ses efforts pour éviter de transporter des jugements de valeur a priori, ceux-ci affleurent toujours. Tous les autres éléments de l'enquête portent avec eux leurs idéologies: l'ancienneté, la rareté et la notoriété, la prise en compte systématique des unica et l'identification, si complexe, des «auteurs» de l'œuvre. Ils ne sont ni objectifs, comme peut l'être la localisation, ni conventionnels, comme l'est la dénomination. En adoptant un classement topographique, l'Inventaire ouvre ses portes à l'idée de patrimoine, dans le sens le plus large. Son classement n'est pas uniquement qualitatif, même si tout inventaire est l'objet d'un « filtrage ». Puisqu'il doit prendre en compte toute œuvre, quelle qu'elle soit, le filtrage ne porte plus sur la qualité relative de chaque œuvre: c'est la catégorie même d'objet artistique ou « patrimonial » qui est en cause.

7 Voulu par Malraux comme un service de recherche, l'Inventaire français est délié de toute finalité partisane. C'est la condition de son efficacité et de sa mission démocratique. La réponse n'est pas donnée avant la question. Le choix n'est pas fait avant l'inventaire. Les goûts et les idéologies patrimoniales sont volatils, et nul ne peut préjuger des choix propres à chaque époque, à chaque communauté. Beaucoup attendent de l'Inventaire un choix entre le bon patrimoine et le mauvais, l'utile et l'inutile. En bref, une sorte de permis de démolir ou un classement de guide touristique. Un tel classement est toujours possible à partir des informations données par l'Inventaire mais il n'a que la valeur que lui donne son auteur et il appartient à chacun de faire le sien. L'Inventaire signale les objets qui méritent d'être protégés, mais il doit aussi permettre de conserver la mémoire de ceux que l'on va détruire. Son irresponsabilité est la condition de sa sincérité. Ce qui ne veut pas dire qu'il est innocent. Le fait de retenir ou non un objet valorise cet objet ou le disqualifie. L'Inventaire est une sorte de « médecine douce » du patrimoine.

La doctrine de la protection ne cesse, depuis 1913, d'élargir son champ d'application : les sites, les «abords» des monuments classés, les secteurs sauvegardés et le récent classement d'ensembles immenses, comme les deux cent cinquante kilomètres du Val de Loire par l'UNESCO. On se contentait de protéger des édifices isolés, on ne se contentera pas longtemps de juxtaposer des périmètres. «Cette construction, écrit Chastel, n'a cessé de perdre sa validité ${ }^{8} »$. Ce dispositif qui tend à banaliser le patrimoine, à en faire la règle et non l'exception, trouve son achèvement dans la loi Solidarité et renouvellement urbain (SRU) du 27 mars 2001 et dans toutes les politiques dites de "développement durable». La protection n'est plus une enclave du passé dans le présent mais un mode de vie présent qui intègre le passé. L'Inventaire général ne fonde pas un patrimoine éternel et immuable, dont la qualité prendrait sa source dans des valeurs universelles et qu'il faut mettre à l'abri du temps, mais sur l'idée que chaque époque, chaque communauté, territoriale ou non, constitue son patrimoine sur ses propres valeurs, qui trouve place dans son époque et évolue avec elle. 


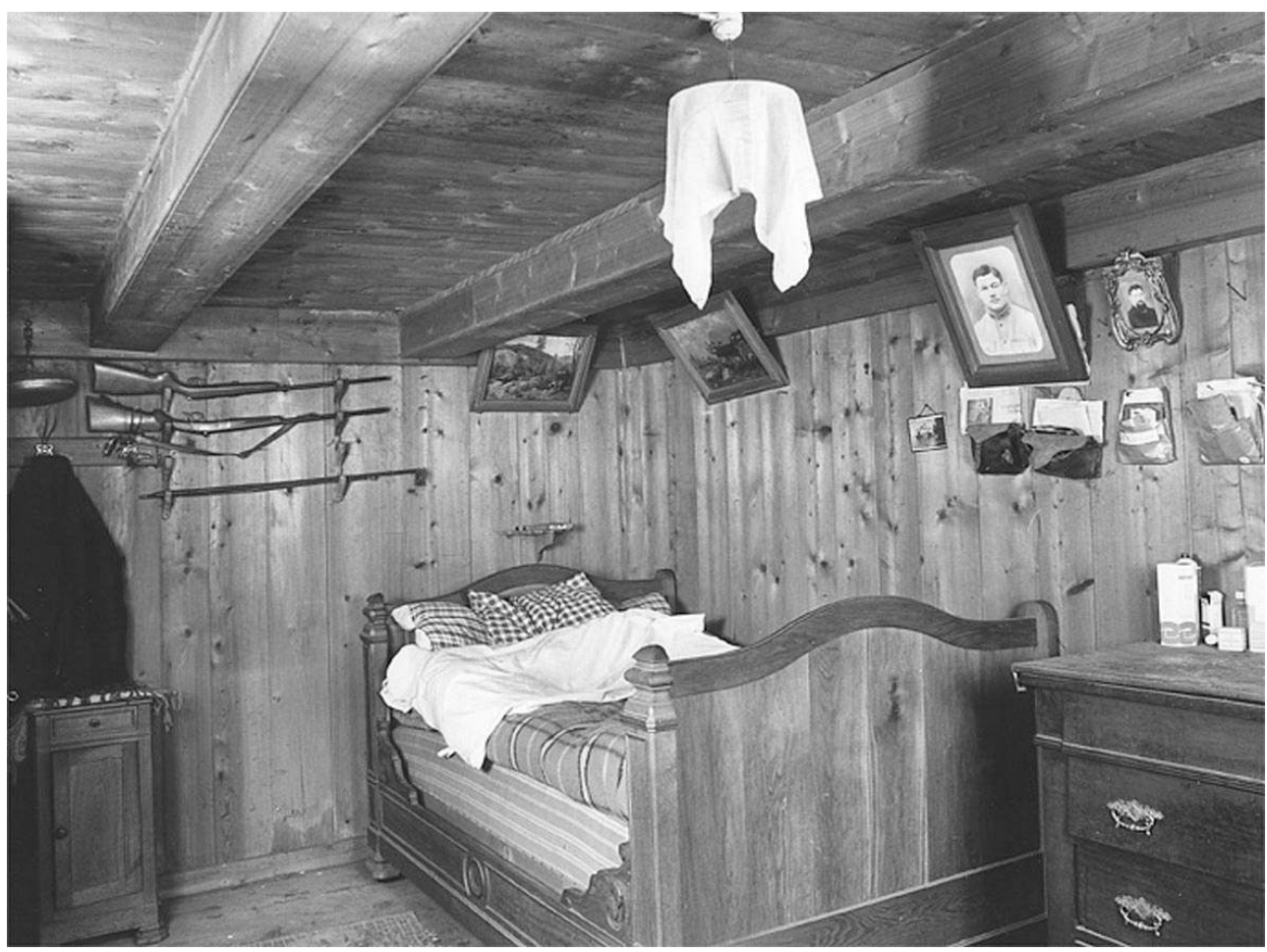

Grand'Combe-Châteleu (Doubs). Chambre de la ferme de Cornabey

Phot. Inv. Y. Sancey (C) Inventaire général, ADAGP, 1977

Il s'agit de bien autre chose que d'un exercice savant ou populaire. Ce que propose l'Inventaire n'est pas le catalogue destiné à abonder les musées de société. C'est le dépassement de l'idée de musée dans un monde qui ne veut plus soustraire les objets à leur environnement mais les intégrer dans notre vie. Pour reprendre la logique du «musée imaginaire », l'Inventaire a surtout « ajouté » aux objets des musées, les maisons d'où elles venaient, les églises par milliers et des villes entières, considérées elles-mêmes comme des œuvres (fig. $\mathbf{n}^{\circ}$ ). Il ouvre un registre qui ne se referme pas, dans lequel chaque objet peut s'inscrire au milieu de tous les autres, y trouver sa raison et sa famille, et prendre la valeur que chacun voudra lui reconnaître. La phrase d'André Chastel citée plus haut montre que c'était bien là pour lui l'enjeu d'un inventaire général: «Le développement de cette enquête exhaustive sur toutes les manifestations de l'art humain aligne assez nettement l'histoire de l'art sur l'histoire des choses...Ce déplacement de l'intérêt comprometil l'attention à la qualité, aux valeurs spécifiques que l'histoire de l'art tendait à retenir pour fondamentales? Cela ne serait possible que si la discipline ne s'adaptait pas à la situation nouvelle, et ne réorganisait pas, à l'occasion de cet extraordinaire élargissement de son domaine, ses concepts et ses instruments. ${ }^{9}$ Et Chastel conclut d'une formule que Malraux aurait pu signer : «Il s'agit pour elle [la discipline] de passer de l'ère du musée à celle de l'inventaire général. Entreprise difficile mais nécessaire."

10 La pensée de Chastel rejoint ici celle d'André Malraux lorsqu'il suggère que l'art a largement pris, depuis la Renaissance, le relais de la religion. C'est l'art, nouvelle église, qui par ses chapelles cimente les groupes virtuels et tisse un fil entre les générations. $\mathrm{Ne}$ peut-on considérer que la notion de " patrimoine ", plus large encore, incluant des objets fonctionnels qui, surtout au moment où ils sont démis de leurs fonctions, apparaissent 
chargés de valeurs symboliques et esthétiques, est en train de se substituer à l'art ou du moins de "l'englober»? Le discours documentaire sobre et méthodique que produit l'Inventaire sur des objets encore hypothétiques, qu'on peut, pour la première fois, considérer ensemble, est adapté à ce nouvel objet qu'on appelle vaguement " patrimoine». Par le seul regard porté sur cet ensemble encore indistinct, il crée la valeur ajoutée sur les objets puis les soumet à ce que les critiques d'art du XVIII ${ }^{e}$ siècle nommaient le «jury secret», celui du public anonyme, celui du marché et des démocraties. La Beauté n'est plus dogmatique : personne n'a le pouvoir d'en imposer les règles. Nous sommes passés, dans le cours du XIXe siècle "par-delà le Beau et le Laid ». Il suffit pour conclure, de citer la dernière phrase du dernier ouvrage sur l'art d'André Malraux qu'il a intitulé L'Intemporel : «Pourquoi l'art ne subirait-il pas une mutation aussi vaste que celle de la Beauté? Nés ensemble, le Musée imaginaire, la valeur énigmatique de l'art, l'intemporel mourront sans doute ensemble. Et l'homme s'apercevra que l'intemporel non plus n'est pas éternel..$^{10}$ ». L'intemporalité, dans laquelle s'inscrit l'objet d'art, prend place dans l'histoire : nous vivons cette époque où le monde urbanisé et industrialisé se cherche un imaginaire, dresse ses monuments, choisit son patrimoine. Voilà longtemps, déjà, que ce monde n'est plus nouveau. Il est temps d'en faire l'inventaire.

\section{NOTES}

1. Chastel, A. Editorial de la Revue de l'art, $n^{\circ}$ 9, 1970.

2. On consultera notamment : Balsamo, I. André Chastel et l'aventure de l'Inventaire. Sciences et conscience du patrimoine, actes des Entretiens du patrimoine (1994), sous la direction de Pierre Nora, Fayard/Editions du patrimoine, 1997, p. 257-267 et Magnien, A. Comment s'est développé l'Inventaire général... Revue administrative, n³03, mai-juin 1998, p. 426-434 et n 304, juilletaoût 1998, p. 543-550. Voir aussi : Melot, M. André Malraux du Musée imaginaire à l'Inventaire général. André Malraux d'un siècle l'autre. Actes du colloque de Cerisy-la-Salle réunis par Jean-Claude Larrat et Jacques Lecarme. Paris: Gallimard, Les cahiers de la NRF, 2002, p. 253-264. Sur les antécédents, voir Massary, X. de. L'Inventaire avant l'Inventaire. Principes, méthode et conduite de l'Inventaire général. Dir. Michel Melot et Hélène Verdier, Editions du patrimoine, 2001.

3. Repris dans: Chastel, A. Architecture et patrimoine, choix de chroniques du journal Le Monde. Imprimerie nationale, 1994, p. 137.

4. Chastel, A. Editorial de la Revue de l'art, $n^{\circ}$ 9, 1970.

5. Sur l'évolution de la notion de patrimoine culturel: Guillaume, Marc. La politique du patrimoine. Galilée, 1980 ; Babelon, J.-P. et Chastel, A. La notion de patrimoine. Revue de l'art, $n$ - 49, 1980, p. 5-32 ; Chastel, A. La notion de patrimoine. Les lieux de mémoire, t.II, Gallimard, 1986, p. 405-450 ; Leniaud, Jean-Michel. L'utopie française, essai sur le patrimoine. Mengès, 1992 ; L'histoire du patrimoine, un essai de périodisation. Patrimoine et société, dir. de JeanYves Andrieux, Presses universitaires de Rennes, 1998; Beghain, Patrice. Le patrimoine : culture et lien social. Presses de Sciences Po, 1998 ; Recht, Roland. Penser le patrimoine. Mise en scène et mise en ordre de l'art. Hazan, 1998. L'Abus monumental ?, actes des « Entretiens du patrimoine » (1998) sous la présidence de Régis Debray, Fayard/Editions du patrimoine, 1999 ; La confusion des monuments, Cahiers de médiologie, coordonné par Michel Melot, n 7, 1999. 
6. Voir les actes des différents « Entretiens du patrimoine».

7. Ce texte fut publié d'abord dans la première plaquette d'information publiée à l'Imprimerie nationale sur l'Inventaire général après sa création le 4 mars 1964. On la retrouve en préface au premier volume d'Inventaire publié, le canton de Carhaix-Plouguer en 1970 puis sur les principales des 900 publications issues de l'Inventaire général.

8. Chastel, A. Editorial de la Revue de l'art, $n^{\circ}$ 9, 1970.

9. Chastel, A. Editorial de la Revue de l'art, $n^{\circ}$ 9, 1970.

10. Malraux, A. L'Intemporel. Paris : Gallimard, 1976, p. 415.

\section{INDEX}

Mots-clés : inventaire, André Chastel, Malraux, patrimoine, tourisme, urbanisme, Monuments Historiques, monument, France, œuvre d'art, recensement, Roger Delarozière, thématique, art, histoire de l'art, site, abord, protection, UNESCO, architecture, objet, industriel

\section{AUTEUR}

\section{MICHEL MELOT}

Conservateur général des bibliothèques honoraire. melotm@ wanadoo.fr 